\title{
ENUMERATION OF CERTAIN SUBGROUPS OF ABELIAN $p$-GROUPS
}

\author{
by I. J. DAVIES
}

(Received 10th May, 1961)

The number of distinct types of Abelian group of prime-power order $p^{n}$ is equal to the number of partitions of $n$. Let $(\rho)=\left(\rho_{1}, \rho_{2}, \ldots, \rho_{r}\right)$ be a partition of $n$ and let $(\mu)=\left(\mu_{1}, \mu_{2}, \ldots, \mu_{s}\right)$ be a partition of $m$, with $\rho_{1} \geqq \rho_{2} \geqq \ldots \geqq \rho_{r}$ and $\mu_{1} \geqq \mu_{2} \geqq \ldots \geqq \mu_{s}, \rho_{i} \geqq \mu_{i}, r \geqq s, n>m$. The number of subgroups of type $(\mu)$ in an Abelian p-group of type $(\rho)$ is a function of the two partitions $(\rho)$, $(\mu)$ and $p$, and has been determined as a polynomial in $p$ with integer coefficients by Yeh (1), Delsarte (2) and Kinosita (3). Their results differ in form but are equivalent.

P. Hall (4) suggested a refinement of this problem in which we require the number of subgroups of type $(\mu)$ in an Abelian p-group of type $(\rho)$ which have a quotient group of type $(\lambda)$. The result, which is a function $g_{\lambda \mu}^{\rho}(p)$ of the three partitions $(\rho),(\lambda),(\mu)$ and $p$, is known to be a polynomial in $p$ of degree $\sum_{i}(i-1)\left(\rho_{i}-\lambda_{i}-\mu_{i}\right)$, and the coefficient of its highest power is the coefficient of the Schur function $\{\rho\}$ in the product of the Schur functions $\{\lambda\}\{\mu\}$. The precise form of the polynomial is however not known in general.

In this note, the polynomial is determined when

$$
\begin{aligned}
& (\rho)=\left(m_{1}^{n_{1}}, m_{2}^{n_{2}}, \ldots, m_{s}^{n_{s}}\right), \\
& (\lambda)=\left(m_{1}^{n_{1}-r_{1}},\left(m_{1}-k\right)^{r_{1}}, m_{2}^{n_{2}-r_{2}},\left(m_{2}-k\right)^{r_{2}}, \ldots, m_{s}^{n_{s}-r_{s}},\left(m_{s}-k\right)^{r_{s}}\right)
\end{aligned}
$$

and

$$
(\mu)=\left(k^{r_{1}+r_{2}+\ldots+r_{s}}\right) \text {, }
$$

where $r_{1}+r_{2}+\ldots+r_{s}=r$. The result is given in the Theorem which is proved by means of the two lemmas which follow.

Lemma 1. The number of subgroups $F$ of type $\left(k^{r}\right)$ in an Abelian p-group $E$ of type $\left(m^{n}\right)$ such that the quotient group E/F is of type $\left(m^{n-r},(m-k)\right)^{r}$, where $r \leqq n, k \leqq m, i s$

where

$$
p^{k r(n-r)} \phi(n, r ; 1 / p)
$$

$$
\phi(s+t, s ; u)=\frac{(1-u)\left(1-u^{2}\right) \ldots\left(1-u^{s+t}\right)}{(1-u) \ldots\left(1-u^{5}\right)(1-u) \ldots\left(1-u^{t}\right)}, \quad s, t>0 .
$$

Proof. From the work of Yeh, Delsarte and Kinosita, it can be shown that the number of subgroups $F$ of type $\left(k^{r}\right)$ in an Abelian $p$-group $E$ of type $\left(m^{n}\right)$ is $p^{k r(n-r)} \phi(n, r ; 1 / p)$. It remains to prove that $E / F$, for every such subgroup $F$, is of the required type.

E.M.S. -A 
Let $E=C_{1} C_{2} \ldots C_{n}$, where $C_{i}$ is a cyclic group of order $p^{m}$, let $F=B_{1} B_{2} \ldots B_{r}$, where $B_{i}$ is a cyclic group of order $p^{k}$, and let $E^{\prime}=C_{1} C_{2} \ldots C_{r}$ with the quotient group $E^{\prime} / F$ isomorphic to a group $F^{\prime}$. We shall need two results.

(i) The quotient group of a cyclic group with respect to a subgroup is also cyclic, and so $C_{i} / B_{i}$ is isomorphic to a cyclic group $D_{i}$ of order $p^{m-k}$.

(ii) If $X, Y$ are any two groups such that $X \cap Y=1$ and, for some group $Z$, the quotient group $Z / X$ is isomorphic to $Y$, then $Z$ is the direct product $X Y$ of $X$ and $Y$.

Now $C_{i}=B_{i} D_{i}$, so that $E^{\prime}=\prod_{1}^{r} C_{i}=\prod_{1}^{r}\left(B_{i} D_{i}\right)=\prod_{1}^{r} B_{i} \prod_{1}^{r} D_{i}=F \prod_{1}^{r} D_{i}$. Since $E^{\prime}$ is also equal to $F F^{\prime}$, we see that $F^{\prime}=\prod_{1}^{r} D_{i}$, i.e. $F^{\prime}$ is isomorphic to the direct product of $r$ cyclic groups $D_{i}$ of orders $p^{m-k}$. Thus $\frac{C_{1} C_{2} \ldots C_{r}}{B_{1} B_{2} \ldots B_{r}}$ is of type $\left((m-k)^{r}\right)$. It follows that $E / F$, which is $\frac{C_{1} C_{2} \ldots C_{r} C_{r+1} \ldots C_{n}}{B_{1} \ldots B_{r}}$, is of type $\left(m^{n-r},(m-k)^{r}\right)$ and the result follows.

Lemma 2. The number of subgroups $F$ of type $\left(k^{r_{1}+r_{2}}\right)$, where $r_{1}+r_{2}=r$, in an Abelian p-group $E$ of type $\left(m_{1}^{n_{1}}, m_{2}^{n_{2}}\right), m_{1}>m_{2}$, such that $E / F$ is of type $\left(m_{1}^{n_{1}-r_{1}},\left(m_{1}-k\right)^{r_{1}}, m_{2}^{n_{2}-r_{2}},\left(m_{2}-k\right)^{r_{2}}\right)$, where $r_{1} \leqq n_{1}, r_{2} \leqq n_{2}, k \leqq m_{2}$, is

$$
p^{k\left[r_{1}\left(N_{1}-R_{1}\right)+r_{2}\left(N_{2}-R_{2}\right)\right]} \phi\left(n_{1}, r_{1} ; 1 / p\right) \phi\left(n_{2}, r_{2} ; 1 / p\right),
$$

where $N_{t}=\sum_{1}^{t} n_{i}, R_{t}=\sum_{1}^{t} r_{i}$

Proof. Let $E$ be generated by $n_{1}$ elements $x_{i}$, each of order $p^{m_{1}}$, and $n_{2}$ elements $y_{j}$, each of order $p^{m_{2}}$. Let $a_{i}=x_{i}^{p^{m_{1}-k}}, i=1,2, \ldots, n_{1}$, and $b_{j}=y_{j}^{p^{m_{2}-k}}$, $j=1,2, \ldots, n_{2}$. Then $a_{i}^{p^{k}}=b_{j}^{p^{k}}=1$. Let the cyclic groups generated by $x_{i}$ and $y_{j}$ be $C_{1 i}$ and $C_{2 j}$ respectively. Every $C_{1 i}$ has one and only one subgroup of order $p^{k}$, namely that generated by $\dot{a}_{i}$, and every $C_{2 j}$ has one and only one subgroup of order $p^{k}$, namely that generated by $b_{j}$. We denote these by $\left[a_{i}\right]$ and $\left[b_{j}\right]$.

The number of subgroups generated by $r_{1}$ of the $a_{i}$ 's is, as in Lemma 1, $p^{k r_{1}\left(n_{1}-r_{1}\right)} \phi\left(n_{1}, r_{1} ; 1 / p\right)$ and the number of subgroups generated by $r_{2}$ of the $b_{j}$ 's is $p^{k r_{2}\left(n_{2}-r_{2}\right)} \phi\left(n_{2}, r_{2} ; 1 / p\right)$. Consider a particular subgroup generated by $r_{2}$ of the $b_{j}$ 's, say the one generated by $b_{1}, b_{2}, \ldots, b_{r_{2}}$. If any of these $b_{j}$ 's is replaced by $b_{j} \times a_{r_{1}+1}^{\alpha_{1}} a_{r_{1}+2}^{\alpha_{2}} \ldots a_{n_{1}}^{\alpha_{n_{1}}-r_{1}}$, where $\alpha_{1}, \ldots, \alpha_{n_{1}-r_{1}}$ have any prescribed values in the range $0,1,2, \ldots, p^{k}-1$, then the group generated by this " augmented "generator is also a cyclic group of order $p^{k}$. The number of these monomials $a_{r_{1}+1}^{\alpha_{1}} a_{r_{1}+2}^{\alpha_{2}} \ldots a_{n_{1}}^{\alpha_{n_{1}}-r_{1}}$ is $p^{k\left(n_{1}-r_{1}\right)}$, since every index $\alpha$ can range from 0 to $p^{k}-1$ and the number of $a_{i}$ 's involved is $n_{1}-r_{1}$. Further, any of these monomials may be used to "augment" any of the $r_{2} b_{j}$ 's and so, in this way, we can construct $p^{k r_{2}\left(n_{2}-r_{2}\right)} \phi\left(n_{2}, r_{2} ; 1 / p\right) \times p^{k r_{2}\left(n_{1}-r_{1}\right)}$ subgroups of 
type $\left(k^{r_{2}}\right)$ and consequently

$$
p^{k\left[r_{1}\left(N_{1}-R_{1}\right)+r_{2}\left(N_{2}-R_{2}\right)\right]} \phi\left(n_{1}, r_{1} ; 1 / p\right) \phi\left(n_{2}, r_{2} ; 1 / p\right)
$$

subgroups of type $\left(k^{r_{1}+r_{2}}\right)$.

It remains to prove that the quotient groups of these subgroups with respect to $E$ are of the type $\left(m_{1}^{n_{1}-r_{1}},\left(m_{1}-k\right)^{r_{1}}, m_{2}^{n_{2}-r_{2}},\left(m_{2}-k\right)^{r_{2}}\right)$ and, further, that there are no other subgroups of $E$ of type $\left(k^{r_{1}+r_{2}}\right)$ having this type of quotient group.

Let $F$ be one of the subgroups of type $\left(k^{r_{1}+r_{2}}\right)$. Without loss of generality, we may take it to be

$$
\left[a_{1}\right]\left[a_{2}\right] \ldots\left[a_{r_{1}}\right]\left[M_{1} b_{1}\right]\left[M_{2} b_{2}\right] \ldots\left[M_{r_{2}} b_{r_{2}}\right],
$$

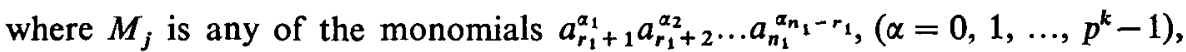
and $M_{j}$ 's in different brackets might possibly be the same. (Note, however, that $\left[M_{j} b_{j}\right]$ and $\left[M_{j} b_{r}\right], r \neq j$, have no elements in common except the identity.)

Then the quotient groups $\frac{C_{1 i}}{\left[a_{i}\right]}, i=1,2, \ldots, r_{1}$, are cyclic of order $p^{m_{1}-k}$, and so, as in Lemma $1, \frac{C_{11} C_{12} \ldots C_{1 r_{1}}}{\left[a_{1}\right]\left[a_{2}\right] \ldots\left[a_{r_{1}}\right]}$ is of type $\left(\left(m_{1}-k\right)^{r_{1}}\right)$.

Since $M_{j}^{p^{k}}=1$, every $M_{j} y_{j}$ generates a cyclic group $C_{2 j}^{\prime}$ of order $p^{m_{2}}$. Thus $\frac{C_{2 j}^{\prime}}{\left[M_{j} b_{j}\right]}$ is cyclic of order $p^{m_{2}-k}$ and it follows that

$$
\frac{C_{11} C_{12} \ldots C_{1 r_{1}} C_{21}^{\prime} C_{22}^{\prime} \ldots C_{2 r_{2}}^{\prime}}{F}
$$

is of type $\left(\left(m_{1}-k\right)^{r_{1}},\left(m_{2}-k\right)^{r_{2}}\right)$. But since $C_{1, r_{1}+1} C_{1, r_{1}+2} \ldots C_{1 n_{1}} C_{21} \ldots C_{2 r_{2}}$ is the same group as $C_{1, r_{1}+1} \ldots C_{1 n_{1}} C_{21}^{\prime} \ldots C_{2 r_{2}}^{\prime}$, we can write $E$ in either of the forms

$$
\begin{aligned}
& C_{11} \ldots C_{1 r_{1}} \ldots C_{1 n_{1}} C_{21} \ldots C_{2 r_{2}} C_{2, r_{2}+1} \ldots C_{2 n_{2}} \\
& C_{11} \ldots C_{1 r_{1}} \ldots C_{1 n_{1}} C_{21}^{\prime} \ldots C_{2 r_{2}}^{\prime} C_{2, r_{2}+1} \ldots C_{2 n_{2}}
\end{aligned}
$$

and so $E / F$ is of type $(\lambda)=\left(m_{1}^{n_{1}-r_{1}},\left(m_{1}-k\right)^{r_{1}}, m_{2}^{n_{2}-r_{2}},\left(m_{2}-k\right)^{r_{2}}\right)$.

To show that there are no other subgroups of $E$ of type $\left(k^{r_{1}+r_{2}}\right)$ having a quotient group of type $(\lambda)$, we note that we are obliged to use $r_{1}$ of the $n_{1}$ $a_{i}$ 's to give the $\left(m_{1}-k\right)^{r_{1}}$ part of $(\lambda)$. We must then choose $r_{2}$ elements of order $p^{k}$ so as to give the $\left(m_{2}-k\right)^{r_{2}}$ part of $(\lambda)$ without affecting the $m_{1}^{n_{1}-r_{1}}$ and $m_{2}^{n_{2}-r_{2}}$ parts. These $r_{2}$ elements must contain a non-vanishing monomial in the $b_{j}$ 's and may contain also a monomial in the $a_{i}$ 's. A monomial in $a_{1}, a_{2}, \ldots, a_{r_{1}}$, say $N_{j}$, will give a group $\left[N_{j} b_{j}\right]$ of order $p^{k}$, but

$$
\left[a_{1}\right] \ldots\left[a_{r_{1}}\right]\left[N_{1} b_{1}\right] \ldots\left[N_{r_{2}} b_{r_{2}}\right]
$$

is nothing more than $\left[a_{1}\right] \ldots\left[a_{r_{1}}\right]\left[b_{1}\right] \ldots\left[b_{r_{2}}\right]$. Hence the only monomials in the $a_{i}$ 's which give distinct subgroups $F$ of the required type are the $M_{j}$ as defined above, which proves the lemma. 
Using these lemmas, we can now prove the main result.

Theorem. If $(\rho)=\left(m_{1}^{n_{1}}, m_{2}^{n_{2}}, \ldots, m_{s}^{n_{s}}\right), m_{1}>m_{2}>\ldots>m_{s}$, and

$$
(\lambda)=\left(m_{1}^{n_{1}-r_{1}},\left(m_{1}-k\right)^{r_{1}}, m_{2}^{n_{2}-r_{2}},\left(m_{2}-k\right)^{r_{2}}, \ldots, m_{s}^{n_{s}-r_{s}},\left(m_{s}-k\right)^{r_{s}}\right) \text {, }
$$

where $r_{1}+r_{2}+\ldots+r_{s}=r, r_{i} \leqq n_{i}(i=1, \ldots, s)$ and $k \leqq m_{s}$, then the number of subgroups $F$ of an Abelian p-group $E$ of type $(\rho)$ which are of type $\left(k^{\left.r_{1}+r_{2}+\ldots+r_{s}\right)}\right.$ and for which $E / F$ is of type $(\lambda)$ is

$$
g_{\lambda, k r}^{\rho}(p)=p^{k \sum_{1}^{s} r_{i}\left(N_{i}-R_{t}\right)} \prod_{i=1}^{s} \phi\left(n_{i}, r_{i} ; 1 / p\right) .
$$

Proof. We assume the result is true for a group $E^{\prime}$ of type $\left(\rho^{\prime}\right)=\left(m_{1}^{n_{1}}, \ldots, m_{t}^{n_{t}}\right)$, $t<s$. We now have to form the direct product of $E^{\prime}$ with $n_{t+1}$ cyclic groups of orders $p^{m_{t+1}}$. Let these be generated by $z_{d}$ where $d=1,2, \ldots, n_{t+1}$. Let $w_{d}=z_{d}^{p^{m_{t+1}-k}}$ so that $w_{d}^{p^{k}}=1$. The number of subgroups of type $\left(k^{r_{t+1}}\right)$ generated by the $w_{d}$ 's is, as in Lemma 1, equal to

$$
p^{k r_{t+1}\left(n_{t+1}-r_{t+1}\right)} \phi\left(n_{t+1}, r_{t+1} ; 1 / p\right) \text {. }
$$

But any $w_{d}$ can be " augmented", as in Lemma 2, by a monomial

$$
a_{r_{1}+1}^{\alpha_{1}} a_{r_{1}+2}^{\alpha_{2}} \ldots a_{n_{1}}^{\alpha_{n_{1}}-r_{1}} b_{r_{2}+1}^{\beta_{1}} b_{r_{2}+2}^{\beta_{2}} \ldots b_{n_{2}}^{\beta_{n_{2}}-r_{2}} \ldots
$$

containing $\left(n_{1}-r_{1}\right)+\left(n_{2}-r_{2}\right)+\ldots+\left(n_{t}-r_{t}\right)$ distinct symbols $a_{i}, b_{j}, \ldots$ with every index $\alpha_{i}, \beta_{j}, \ldots$ capable of any of the values from 0 to $p^{k}-1$. Hence the number of subgroups of type $\left(k^{r_{t+1}}\right)$ which we can construct from the " augmented" $w_{d}$ 's is

$$
p^{k r_{t+1}\left(N_{t+1}-R_{t+1}\right)} \phi\left(n_{t+1}, r_{t+1} ; 1 / p\right) .
$$

As in Lemma 2, the quotient group is of type $\left(m_{1}^{n_{1}-r_{1}},\left(m_{1}-k\right)^{r_{1}}, \ldots\right.$, $\left.m_{t+1}^{n_{t+1}-r_{t+1}},\left(m_{t+1}-k\right)^{r_{t+1}}\right)$ and there are no further subgroups possible under the conditions for $F$ prescribed in Lemma 2.

The theorem now follows by induction.

\section{REFERENCES}

(1) Y. YeH, On prime power Abelian Groups, Bull. Amer. Math. Soc., 54 (1948), 323-327.

(2) S. Delsarte, Fonctions de Möbius sur les groupes abeliens finis. Ann. of Math., (2) 49 (1948), 600-609.

(3) Y. KINOSITA, On an enumeration of certain subgroups of a $p$-group. $J$. Osaka Inst. Sci. Tech. Part I, 1 (1949), 13-20.

(4) P. Hall, Edinburgh Mathematical Society Colloquium, St Andrews, 1955.

UNIVERSITY COLLEGE

SWANSEA 